\title{
Dimensional reduction for the inverse problem of neural field theory
}

\author{
Roland Potthast ${ }^{1}$ and Peter beim Graben ${ }^{2 *}$ \\ 1 Department of Mathematics, University of Reading, Reading, UK \\ 2 School of Psychology and Clinical Language Sciences, University of Reading, Reading, UK
}

Edited by:

Hava T. Siegelmann, University of

Massachusetts Amherst, USA

Reviewed by:

Axel Hutt, INRIA CR Nancy, France

Thomas Wennekers, University of

Plymouth, UK

${ }^{*}$ Correspondence:

Peter beim Graben, School of

Psychology and Clinical Language

Sciences, University of Reading,

Whiteknights, PO Box 217, Reading

RG6 6AH, UK.

e-mail: p.r.beimgraben@reading.ac.uk
Inverse problems in computational neuroscience comprise the determination of synaptic weight matrices or kernels for neural networks or neural fields respectively. Here, we reduce multidimensional inverse problems to inverse problems in lower dimensions which can be solved in an easier way or even explicitly through kernel construction. In particular, we discuss a range of embedding techniques and analyze their properties. We study the Amari equation as a particular example of a neural field theory. We obtain a solution of the full 2D or 3D problem by embedding OD or 1D kernels into the domain of the Amari equation using a suitable path parametrization and basis transformations. Pulses are interconnected at branching points via path gluing. As instructive examples we construct logical gates, such as the persistent XOR and binary addition in neural fields. In addition, we compare results of inversion by dimensional reduction with a recently proposed global inversion scheme for neural fields based onTikhonov-Hebbian learning. The results show that stable construction of complex distributed processes is possible via neural field dynamics. This is an important first step to study the properties of such constructions and to analyze natural or artificial realizations of neural field architectures.

Keywords: neural field theory, Amari equation, synaptic weight kernels, inverse problems, Tikhonov-Hebbian learning, path embeddings, Hilbert space dynamics

\section{INTRODUCTION}

Neural field theories are continuum approximations for highdimensional neural networks (Griffith, 1963; Wilson and Cowan, 1973; Amari, 1977; Ermentrout and McLeod, 1993; Jirsa and Haken, 1996; Robinson et al., 2001; beim Graben, 2008). In a rather general form (Wilson and Cowan, 1973; beim Graben, 2008), the neural field equation can be given by an integro-differential equation

$\tau(x) \frac{\partial u(x, t)}{\partial t}+u(x, t)=$

$\int_{-\infty}^{t} \int_{D} w\left(x, x^{\prime}\right) G\left(t-t^{\prime}\right) f\left[u\left(x^{\prime}, t^{\prime}-\frac{\left|x-x^{\prime}\right|}{c}\right)\right] d t^{\prime} d x^{\prime}$,

where $u(x, t)$ describes the spatio-temporal activity of the neural tissue at position $x \in D$ and at time $t \in \mathbb{R}$. The spatial domain $D \subset \mathbb{R}^{n}$ with $n=1,2$ or 3 are typical dimensions in applications. The function $\tau(x)$ describes a spatially dependent time constant for neural activation, while the integral kernel $w\left(x, x^{\prime}\right)$ indicates the synaptic connectivity and strength between sites $x$ and $x^{\prime}$ in the neural tissue. The temporal kernel $G(t)$ is the postsynaptic impulse response function, and $c$ the neural propagation velocity. Finally, $f$ is a sigmoidal activation function describing the conversion of membrane potential $u(x, t)$ to spike rates at the axon hillock.

The interpretation of Eq. 1 is therefore as follows: The lefthand side describes the intrinsic decay dynamics of neural activation $u(x, t)$ with time constant $\tau(x)$, whereas the right-hand side accounts for the net input to $u(x, t)$ via synaptic connections. The spatial integral generalizes the usual weighted summation of activity delivered to neurons at $x$ from neurons at $x^{\prime}$. On the other hand, the temporal convolution models summation of postsynaptic potentials over time together with axonal propagation delay.

Note that even more general versions of neural field equations contain a distribution of propagation velocities $\rho(c)$, instead of a fixed velocity $c$. Accordingly, they involve a third integration over $d c$ at the r.h.s. of the neural field equation (Nunez, 1995; Hutt and Atay, 2006). Hutt and Atay (2006) were able to demonstrate that the variance of the velocity distribution affects the frequency of bifurcating periodic solutions and the phase speed of travelling waves. More interestingly, they discovered that the introduction of velocity distributions lead to the maximization of travelling front speed.

Equation 1 can be substantially simplified by assuming spatial homogeneity of time constants, $\tau(x)=\tau$, infinite signal propagation $c=\infty$ and instantaneous postsynaptic processing, $G(t)=\delta(t)$. As a result, one obtains the Amari equation (Amari, 1977) with inhomogeneous kernel in the form

$\tau \frac{\partial u(x, t)}{\partial t}+u(x, t)=\int_{D} w(x, y) f(u(y, t)) d y, x \in D, t>0$

where the nonlinear activation function $f$ is given as

$f_{\eta, \beta}(z)=\frac{1}{1+e^{-\beta(z-\eta)}}$

with constants gain $\beta>0$, and threshold $\eta \in \mathbb{R}$. The Amari equation characterizes a simple neural field theory and serves as a basic model to discuss and implement neural field architectures and neural field phenomena. We shall restrict our attention to basic important phenomena here. 
Interpreting the domain $D \subset \mathbb{R}^{n}$ of the Amari equation 2 not as a physical substrate realized by actual biological neurons, but rather as an abstract feature space for computational purposes, one also speaks about dynamic field theory (Thelen et al., 2001; Erlhagen and Schöner, 2002).

The description of logical reasoning and digital computation by means of neural network architectures is at the focus of research since the pioneering work of McCulloch and Pitts in the 1940s (McCulloch and Pitts, 1943) and has been investigated, e.g. by Balkenius and Gärdenfors (1991), Mizraji and Lin (2001), Blutner (2004), in the sequel. In the framework of neural or dynamic field theory, Wennekers $(2006,2007)$ used operational cell assemblies as such a computing paradigm. Here, we will study another approach to this goal on the basis of neural field theory. The general task of implementing cognitive or logical processes can be described by a hierarchy of three tiers (compare beim Graben and Potthast 2009). The top level is the cognitive level where cognitive processes are modelled with tools from symbolic logic. The second level is the representation level, which contains fields representing the cognitive processes in terms of wave fields, states and field dynamics. The third and bottom level is a neural field level where neural fields and neural kernels implement the dynamics of the representation level.

When the dynamical fields in the representation level are given, we need to solve the inverse problem of constructing appropriate neural kernels implementing the dynamics, compare beim Graben and Potthast (2009), Potthast and beim Graben (in press). Usually, for more complex tasks of cognition these are high-dimensional inverse problems, which exhibit significant computational cost as well as high degree of instability or ill-posedness.

In this work, we mainly address two tasks. The first task is to provide a method for constructing neural field kernels for more complex cognitive tasks which is less computationally expensive and less ill-posed than the earlier approaches (beim Graben and Potthast, 2009; Potthast and beim Graben, in press).

Usually the term problem reduction is used when a difficult problem is solved by reducing it to easier problems. In this sense the term dimensional reduction describes the process from an inverse problems perspective, since we reduce the multi-dimensional inverse problems to the solution of low-dimensional tasks.

We will solve the problem by constructing low-dimensional embeddings of neural kernels into higher-dimensional neural domains. In particular, we will describe zero-dimensional and onedimensional embeddings into two- (or possibly three-dimensional) neural tissue. This is employed for the construction of paths for neural pulses, for gluing different path patches together, for designing AND, OR and XOR gates as well as delay gates which can be used for synchronization of neural pulses.

The second task is to study more general Hilbert-space based implementations of cognitive processes on the basis of neural field theory. Neural field equations such as Eq. 1 or the Amari equation 2 are often transformed into partial differential equations for further analysis (Jirsa and Haken 1997; Coombes et al., 2003). This, however, restricts the synaptic weight kernels considerably, by presupposing local interactions. On the other hand, generating and understanding non-local distributed cognitive dynamics with complex logical decisions is of high interest both for controlling and constructing neural computing architectures as well as for analyzing natural phenomena in biological neural systems (Jirsa and Kelso 2000; Bressloff and Cowan 2002). We will realize this dynamics by using basis transformations from special embedded dynamics, in particular we employ basis transformations from simple localized basis functions onto distributed global functions on our neural domain which are realized as vectors with random components.

We will realize some generic logical tasks like AND, OR, XOR and more complex tasks like binary addition with three binary inputs and a gating pulse on the basis of neural field theory. To this end we first design the processes on an abstract logical level and then describe different realizations and embeddings in the framework of a neural field theory.

In Section "Solving Inverse Neural Field Problems" we provide an introduction into main tools from the theory of inverse problems which can be used to solve low or high-dimensional inverse problems. We will use the tools in subsequent sections for constructing low-dimensional neural field kernels for prescribed neural dynamics. Section "Embedding One-dimensional Processes into 2d or $3 \mathrm{~d}$ Neural Tissue" is the first key section to describe embedding techniques and to construct basis kernels for elementary logical functions based on a neural field environment. Section "Embedding 0D Logic into Neural Tissue" serves to describe a zero-dimensional version of the embedding theory. This can also be viewed as an embedding of a classical neural network into a neural field environment. An abstract view based on Hilbert space theory is provided in Section "An Abstract View and Basis Transformations". We discuss Hilbert space based dynamics and basis transformations in the framework of Amari neural fields with a particular focus on the stable construction of global neural dynamics. This leads to a distributed non-local logical dynamics, which is described and implemented. Numerical examples for each of the above sections are provided.

\section{SOLVING INVERSE NEURAL FIELD PROBLEMS}

This section serves as summary and introduction into solving inverse problems in the framework of the Amari equation 2 as carried out by beim Graben and Potthast (2009), Potthast and beim Graben (in press) and Potthast and beim Graben (in press). It will be employed many times for the one-dimensional pulse construction in the following sections.

We prescribe one or several complete time-dependent patterns $v_{\xi}(x, t), \xi=1, \ldots, n$ for $x \in D, t \geq 0$ with some domain $D \subset \mathbb{R}^{p}$. For our further discussion we assume that the nonlinear activation function $f: \mathbb{R} \rightarrow[0,1]$ is known. Then, we search for kernels $w(x, y)$ for $x, y \in D$, such that the solutions of the Amari equation with initial conditions $u(x, 0)=v_{\xi}(x, 0)$ satisfies $u(x, t)=v_{\xi}(x, t)$ for $x \in D$, $t \geq 0$, and $\xi=1, \ldots, n$.

As a first step, we transform Eq. 2 into a linear integral equation. Defining

$\varphi_{\xi}(x, t)=f\left[v_{\xi}(x, t)\right], x \in D, t \geq 0$,

$\psi_{\xi}(x, t)=\tau \frac{\partial v_{\xi}(x, t)}{\partial t}+v_{\xi}(x, t), \quad x \in D, \quad t \geq 0$,

and employing the integral operator 
$(W \varphi)(x)=\int_{D} w(x, y) \varphi(y) d y, \quad x \in D$,

leads to a reformulation of the inverse problem into the equation

$\psi_{\xi}(x, t)=W \varphi_{\xi}(x, t), x \in D, t \geq 0, \xi=1, \ldots, n$,

where here the kernel $w(x, y), x, y \in D$, of the linear integral operator $W$ is unknown. Equation 7 is linear in the kernel $w$. It can be rewritten as

$\Psi=W \Phi$

with

$\Phi=\left(\varphi_{1}, \ldots, \varphi_{n}\right), \Psi=\left(\psi_{1}, \ldots, \psi_{n}\right)$.

For every fixed $x \in D$ we can rewrite Eq. 7 as

$\psi_{\xi, x}(t)=\int_{D} \varphi_{\xi}(y, t) w_{x}(y) d y \quad t \geq 0$

with

$w_{x}(y)=w(x, y), x, y \in D, \psi_{\xi, x}(t)=\psi_{\xi}(x, t), x \in D, t \geq 0$.

If $\varphi$ is continuous in $y$ and $t$, then for fixed $x$, Eq. 10 is a Fredholm integral equation of the first kind with continuous kernel $\varphi$. This equation is known to be ill-posed (Kress, 1989), i.e. we do not have (a) existence or (b) uniqueness in general and even if we have uniqueness the solution does not depend in a (c) stable way on the right-hand side.

In order to cope with ill-posedness and instability, several regularization techniques have been proposed in the literature. Among them, Tikhonov regularization is a very general scheme which can be derived via three different approaches: (i) by a spectral approach, (ii) from matrix algebra, or (iii) as an optimization procedure for solving an ill-posed equation $V g=f$. Clearly, a solution to the equation $V g=f$ can be searched by the minimization

$\min _{g \in X}\|V g-f\|$

where $X$ denotes some appropriate Hilbert space $X$, for example the space $L^{2}(D)$ of square integrable functions on some domain $D$. The normal equations (compare Kress, 1999) for the minimization problem are given by

$V^{\star} V g=V^{\star} f$.

The operator $V^{\star} V$ does not have a bounded inverse. Stabilization is reached by adding a small multiple of the identity operator $I$ (Kress, 1999), i.e. by solving

$\left(\alpha I+V^{\star} V\right) g=V^{\star} f$,

which corresponds to adding a stabilization term $\alpha\|g\|^{2}$ to the minimization (Eq. 11), leading to the third form of the Tikhonov regularization

$\min _{g \in X}\left(\|V g-f\|^{2}+\alpha\|g\|^{2}\right)$.

The operator Eq. 12 is usually discretized by standard procedures and then leads to a matrix equation which can be solved either directly or by iterative methods (Kress, 1999).
The Moore-Penrose pseudoinverse is given by the limit of the Tikhonov regularization for $\alpha \rightarrow 0$, i.e. it is

$V^{\dagger}=\left(V^{\star} V\right)^{-1} V^{\star}=\lim _{\alpha \rightarrow 0}\left(\alpha I+V^{\star} V\right)^{-1} V^{\star}$.

However, as discussed above this limit will lead to satisfactory reconstructions only for well-posed problems. For the abovementioned ill-posed inverse problem we have to employ $\alpha>0$.

These techniques were applied to neural pulse construction problems by Potthast and beim Graben (in press), Potthast and beim Graben (in press) and beim Graben and Potthast (2009). In particular, the authors demonstrated the feasibility of regularized construction techniques for synaptic kernel construction. Properties of the solutions were investigated and the ill-posedness of the problem was proven and demonstrated by particular examples.

Challenges for multi-dimensional inverse problems. As a preparation to the next parts of this work here we discuss one major disadvantage of the above approach when more complex problems are addressed.

It is well-known that solving multi-dimensional inverse problems is a difficult and challenging task of current research (Colton and Kress, 1998). In particular, it is well-known that in general the ill-posedness of an inverse problem increases if

I. we use higher discretizations for the simulations of the forward problem,

II. the dimension and complexity of the mapping under consideration increases.

The reason for this phenomenon in both cases is that the calculations take into account more singular values and singular functions. The singular values strongly decay and taking more singular values into account corresponds to larger instability.

For cognitive neuroscience this means that both increasing the dimension and complexity of the simulated dynamics as well as enhancing the simulation algorithms by higher discretization will cause more severe instabilities for kernel construction problems. In addition, the computational costs are strongly dependent on the size of the problem under consideration, measured in terms of

a. the dimension of the neural domain,

b. the complexity of the dynamics,

c. the number of time discretization points,

d. the spatial discretization of the problem,

where c) and d) usually depend to some extent on a) and b), for example discretizations of a multi-dimensional problem needs much more discretization points than discretization of a onedimensional problem.

Here, the size and condition number of the matrix $\Phi$ defined in Eq. 9 will strongly increase with the number of time-discretization steps. A detailed discussion of these effects can be found in Potthast and beim Graben (in press) and Potthast and beim Graben (in press). Yet the above works do not propose any approach to resolve the problems. They focus on the study of simple problems with basic tools from the theory of inverse problems. Our goal here is to introduce a computational technique which resolves the issue by use of embedding techniques. Our approach is related to similar approaches such as operational cell assemblies and continuous 
attractor networks (Thelen et al., 2001; Erlhagen and Schöner, 2002; Wennekers, 2006, 2007) and we expect that the algorithms developed here can be employed to such approaches as well. The techniques allow stable and efficient solutions to complex and distributed neural modelling problems, we expect it to be an important step towards addressing more realistic questions of cognitive dynamics.

\section{EMBEDDING ONE-DIMENSIONAL PROCESSES INTO 2D OR 3D NEURAL TISSUE}

The approach in this work is to solve low-dimensional inverse problems with mild or no instability and to embed the solution into a higher-dimensional space. To this end we will first introduce an embedding technique in the next subsection and then apply it to plain pulse kernels and delay kernels. We show how different paths can be linked together leading to different types of branching point dynamics. Then, the construction of simple and more complex logical functions on the basis of embedded pulses is described.

The embedding technique here is independent of the technique which is used to calculate the kernels which are embedded. We will flexibly employ two different approaches to calculate low-dimensional kernels which are then embedded into higher-dimensional neural domains:

(1)analytical solutions/kernels given explicitly in closed form or

(2) kernels constructed numerically by solving the inverse problem of Section "Solving Inverse Neural Field Problems" on a low-dimensional set.

\section{PATH EMBEDDING}

Let $\tilde{w}(s, \tilde{s})$ for $s, \tilde{s} \in[a, b]$ be a one-dimensional neural kernel. Our neural tissue is given by a domain $D \subset \mathbb{R}^{2}$. We consider a path $\gamma:[a, b] \rightarrow D$ given by parametrization $s \mapsto \gamma(s)$, and define its trace by

$\Gamma:=\{\gamma(s): s \in[a, b]\}$.

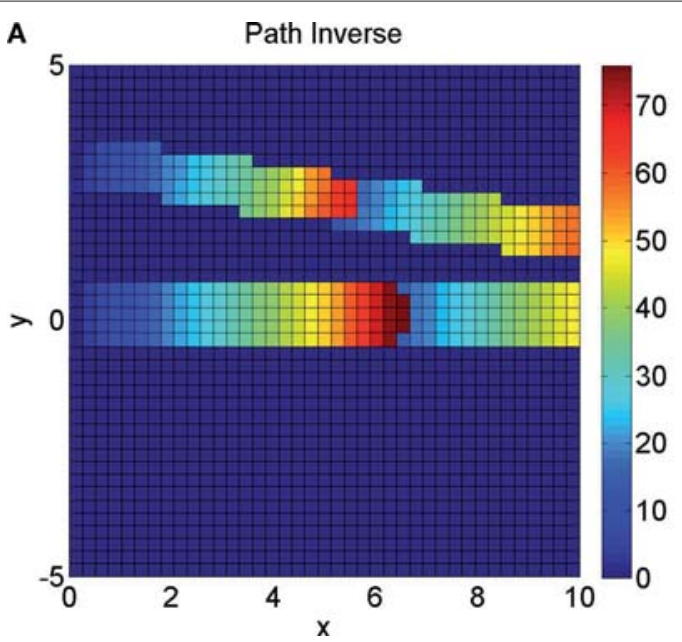

FIGURE 1 | The image (A) shows several path embeddings, where for a point $x \in D$ in the neural tissue $D$ we visualize the index of the path vector $\boldsymbol{\Gamma}$ which is mapped onto $\boldsymbol{x}$. The colour in $(\mathbf{A})$ shows the parameter of a particular path. Where red overlaps light blue, two paths patches are glued together. The embedded kernel (calculated by aTikhonov-Hebbian
We will use $|\cdot|$ for the Euclidean norm in $\mathbb{R}^{n}$ and $d(x, G)$ for the Euclidean distance of a point $x$ to the domain or set $G$. We assume that

A1. $\gamma$ is differentiable, i.e. for every point $x \in \Gamma$ there is a tangential vector $\zeta(x) \in \mathbb{R}^{2}$ and a normal vector $\mathrm{v}(x) \perp \zeta(x)$,

A2. the size of the derivative $\gamma^{\prime}(s)$ is strictly positive, i.e. there is a constant $c_{1}$ such that $\left|\gamma^{\prime}(s)\right| \geq c_{1}>0$ for all $s \in[a, b]$,

A3. the mapping $s \mapsto \gamma(s)$ is injective, i.e. there are no crossing points and double points of the path,

A4. the path embedding has sufficient distance to the boundaries of our neural tissue, i.e. $d(\Gamma, \partial D) \geq c_{2}>0$ with some sufficiently large constant $c_{2}$.

The last condition is convenient to avoid technical overload of the arguments below. We are then prepared to define a tube of diameter $\rho>0$ around a path $\Gamma$ by

$\Gamma_{\rho}:=\{x \in D: d(x, \Gamma) \leq \rho\}$

where $d$ denotes the distance from $x$ to $\Gamma$. If $\rho$ is sufficiently small, the tube $\Gamma_{\rho}$ can also be defined as the set

$\Gamma_{\rho}=\{x+r v(x): x \in \Gamma,|r|<\rho\}$.

In this case, for $x \in \Gamma_{\rho}$ there is a unique pair of parameters $(s, r)=T(x)$ such that $x=\gamma(s)+r v[\gamma(s)]$. We use the abbreviation $\gamma_{\rho}(s, r):=\gamma(s)+r v[\gamma(s)]$ for $s \in[\mathrm{a}, \mathrm{b}]$ and $r \in[-\rho, \rho]$.

Definition 3.1. (Kernel Embedding) For $\rho$ sufficiently small as in Eq. 17 depending on $c_{2}$ and $\Gamma$ we define the embedding of $\tilde{w}$ into $D$ by

$w(x, y):= \begin{cases}\frac{\tilde{w}(s, \tilde{s})}{2 \rho\left|\gamma^{\prime}(s)\right|} & x, y \in \Gamma_{\rho},(s, r)=T(x),(\tilde{s}, \tilde{r})=T(y) \\ 0 & \text { otherwise. }\end{cases}$

An example of such a kernel embedding is shown in Figure $\mathbf{1 .}$

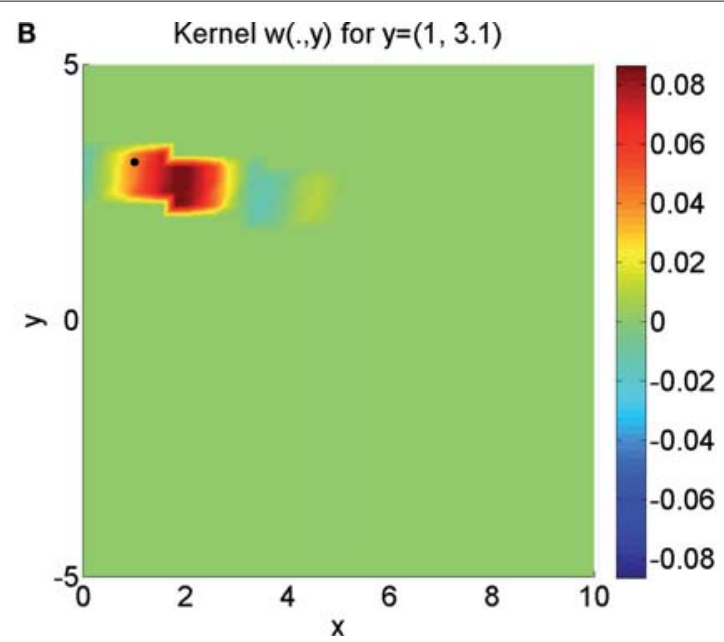

approach described in Section "Solving Inverse Neural Field Problems") for the first of these path and a point $y=(2.4276,2.5724)$ is shown in image (B), illustrating the embedded kernel (Eq. 18). Here, the neuron located at the black point is exciting the red area and inhibiting blue areas. 
We later study the processing of pulses similar to the work shown in beim Graben and Potthast (2009), Potthast and beim Graben (in press) and Potthast and beim Graben (in press). In particular, later we use Gaussian pulses

$v(x, t):=e^{-\sigma \cdot(x-c t)^{2}}, x, t \in \mathbb{R}$,

with $\sigma>0$ and speed $c>0$. With the embedded kernel under the conditions A1-A4 we will obtain a two-dimensional pulse which is analogous to the one-dimensional pulse dynamics, but now follows the prescribed path $\Gamma$ or tube $\Gamma_{\rho}$, respectively.

The construction of the two-dimensional kernels (Eq. 18) is carried out such that the kernel is the same for all points in a tube which are on a line

$L(x):=\{x+r v(x):|r|<\rho\}$.

This also leads to an equivalence of the neural dynamics generated by the one-dimensional kernel and the two-dimensional embedded kernel (18).

We call a function $u_{0}$ embedded into $\Gamma_{\rho}$, if it is constant on lines $L(x)$ defined by Eq. 20 for all $x \in \Gamma$. In this case $u_{0}$ corresponds to a one-dimensional function $\tilde{u}_{0}$ defined on the parametrization interval $[a, b]$ of $\Gamma$ such that $u_{0}$ is the embedded version of $\tilde{u}_{0}$.

Theorem 3.2. Consider a path embedding $\Gamma$ which satisfies A1-A4 and let the initial value $u_{0}$ be an embedded function from $\tilde{u}_{0} \in C([a, b])$ according to Definition 3.1. Then, the dynamics for the embedded kernel $w$ with initial value $u_{0}$ on $\Gamma$ and the dynamics of the one-dimensional kernel $\tilde{w}$ with initial values $\tilde{u}_{0}$ are equivalent, i.e. the diagram

$$
\begin{array}{ccl}
\tilde{u}_{0} & \text { 1D dynamics } & \tilde{u}(\cdot, t) \\
\text { embedding } \downarrow & \rightarrow & \downarrow \text { embedding } \\
u_{0} & \text { 2D dynamics } & u(\cdot, t)
\end{array}
$$

is commutative, i.e. we can either first carry out the one-dimensional dynamics and then embed the resulting dynamical field or we can first embed the initial condition and then carry out the embedded two-dimensional dynamics.

Proof. The proof is a direct consequence of the particular embedding technique. The two-dimensional dynamics includes the two-dimensional integral with the excitation or forcing term

$$
F(x):=\int_{D} w(x, y) f(u(y, t)) d y, x \in D
$$

We evaluate this integral for points $x=\gamma_{\rho}(s, r), s \in[a, b]$ and $r \in[-\rho, \rho]$ in the tube $\Gamma_{\rho}$ as follows. Substituting $d y=\left|\gamma^{\prime}(\tilde{s})\right| d \tilde{s} d \tilde{r}$ for $\tilde{F}(s, r):=F\left(\gamma_{\rho}(s, r)\right)$ we calculate

$$
\begin{aligned}
\tilde{F}(s, r) & =\int_{D} w\left(\gamma_{\rho}(s, r), y\right) f(u(y, t)) d y \\
& =\int_{a}^{b} \int_{-\rho}^{\rho} \frac{\tilde{w}(s, \tilde{s})}{2 \rho\left|\gamma^{\prime}(\tilde{s})\right|} f(u(\tilde{s}, t))\left|\gamma^{\prime}(\tilde{s})\right| d \tilde{r} d \tilde{s} \\
& =\int_{a}^{b} \tilde{w}(s, \tilde{s}) f(u(\tilde{s}, t)) d \tilde{s}
\end{aligned}
$$

which is the r.h.s of the one-dimensional Amari equation, thereby reducing the two-dimensional dynamics to a onedimensionalcase.

\section{PLAIN PULSES}

The goal of this section is to set up simple kernels which lead to onedimensional travelling neural pulses $v(x, t)$ for $x \in \mathbb{R}$ and $t \in \mathbb{R}$ on the real line $\mathbb{R}$. The generation of neural pulses by homogeneous kernels has been intensely studied, compare Amari (1977), Ermentrout and McLeod (1993), Coombes et al. (2003), Potthast and beim Graben (in press). Here, we will either provide an analytical solution or employ the Tikhonov-Hebbian approach described in Section "Solving Inverse Neural Field Problems". We apply the techniques of the preceding Section "Solving Inverse Neural Field Problems" to construct a kernel to generate the dynamics of a pulse of the form (Eq. 19).

Alternatively, we may obtain stable pulse-like solutions as follows. We define a one-dimensional kernel with backward Gaussian inhibition and forward Gaussian excitation by explicit analytical construction

$\tilde{w}(s):=c_{+} e^{-\sigma \cdot\left(s-s_{0}\right)^{2}}-c_{-} e^{-\sigma \cdot\left(s+s_{0}\right)^{2}}, s \in \mathbb{R}$

with constants $c_{+}, c_{-}>0$ and $s_{0}>0$. For an appropriate choice of constants $c_{ \pm}$and $s_{0}$ by $w(x, y):=\tilde{w}(x-y)$ and initial conditions by

$v(x, 0)=e^{-\sigma_{0} x^{2}}, x \in \mathbb{R}$,

we obtain travelling pulses in one dimension for the Amari field equation 2 . This can be verified by numerical simulation or, for particular cases, by elementary arguments. We skip the details of the arguments since they work along the lines of Potthast and beim Graben (in press).

\section{DELAY GATES}

Delay gates are logical elements which synchronize pulses travelling with different speed or which have to travel over a different distance. A delay gate keeps a pulse active over some time interval, such that pulses arriving earlier and pulses arriving later as input into some logical element lead to simultaneous excitation of the input areas. Delay gates are neural field implementations of delay lines for neural networks (Hertz et al., 1991).

Here, we will construct delay gates which realize an excitation at the exit of the gate as soon as a pulse enters the gate area and keep the excitation at the exit constant while the pulse is passing through the delay gate. This synchronizes pulses over a time window of the travel time of a pulse through the gate.

In more detail, a delay gate is a kernel $w(x, y)$ defined on an interval $[a, b] \subset \mathbb{R}$ such that any pulse with sufficiently large active support entering the interval $[a, b]$ leads to an excitation of the neural field in a neighbourhood of sufficient size of $b$ as long as the pulse is travelling through the interval $I_{[a, b]}$.

The construction of delay gates can be done by solving neural inverse problems or by direct construction. Here, we employ the inversion technique of Section "Solving Inverse Neural Field Problems". A delay gate is obtained by solving the inverse problem (Eqs. 7-10) with training pulse given by

$v(x, t):=e^{-\sigma \cdot\left(x-\frac{(c t-a)}{c T-a}\right)^{2}}+f_{\eta_{0}, \beta}(t) \cdot e^{-\sigma(x-b)^{2}}, x \in[a, b]$ 
for $t \in[0, T]$, where $f_{\eta_{0}, \beta}$ denotes the sigmoidal activation function defined in Eq. 3. The constants $\eta_{0}$ and $\beta$ control the time delay after which the excitation at the exit point of the delay gate becomes active.

Without an initial field the field in the gate will just be 0 . If a pulse enters the gate, it corresponds to the initial training pattern (Eq. 28) and excites the complete path of the training pattern, which leads to an excitation of the exit pulse after a time interval $\eta_{0}$ which is kept constant for the duration of pulse travel through the gate.

We numerically demonstrate a delay gate in Figure 2. The figure shows one time slice of the dynamics for two different pulses. In the upper image the pulse entered the gate earlier than in the lower image, both pulses simultaneously excite a pulse at the exit area around coordinate $x=10$.

\section{BRANCHING POINTS AND GLUING PATHS TOGETHER}

We employ the following technique for branching points and gluing paths together. As a preparation we note that the kernel for a plain path provides a direction for this path. A pulse has an entry point at one side of the tube and an exit point at the other. Pulses will only propagate from the entry point to the exit point. A pulse entering an exit point will die.

We speak of gluing together two or more paths at a point when we overlap an exit area with one or several entry areas or an entry area with several exit areas of different paths. We have employed the following elementary assumption for our constructions:

A5. We assume that there is a positive distance between any two tubes given by two paths, except for overlap in the case where we glue together two or more paths at a branching point.

The following points describe the gluing process in more detail:

1. If we want to glue together two paths $\Gamma_{1}$ and $\Gamma_{2}$, they need to be embedded with sufficient overlap such that a pulse exciting the tube $\Gamma_{1, \rho}$ (which does not have neural connections from the interior of $\Gamma_{1, \rho}$ into the exterior of $\Gamma_{1, \rho}$ ) excites the pulse travelling through the tube $\Gamma_{2, \rho}$. Here, we used an overlap as described in A5. containing a ball of radius $\rho$.

2. Consider the embedding $w_{1}$ for path $\Gamma_{1}$ and the embedding $w_{2}$ for path $\Gamma_{2}$, with overlap constructed as demanded in Item 4 . Then a glued pulse is constructed by

$w(x, y):=w_{1}(x, y)+w_{2}(x, y), x, y \in D$.

3. According to the embedding technique for kernels the intersection of the support of $w_{1}$ and $w_{2}$ is a subset of the path overlap, i.e. where both $x$ and $y$ are in $\Gamma_{1} \cap \Gamma_{2}$. For most kernels considered in this work the kernels do not have a significant size when $x$ and $y$ are very close to each other, such that the summation (Eq. 29) does not disturb the pulse behaviour significantly in $\Gamma_{1} \cap \Gamma_{2}$.

We demonstrate the gluing of paths in Figure 1. Our numerical tests with travelling pulses below confirm the above arguments and show that we can glue paths together without difficulty. The technique has been employed for the example of binary addition in Section "An Abstract View and Basis Transformations", compare also Figure 5.

Branching and OR Logic. Branching points are points where more than two pulses are glued together. Given three paths $\Gamma_{1}, \Gamma_{2}$ and $\Gamma_{3}$ we construct a branching point from embeddings $w_{1}, \ldots, w_{3}$ which have some overlap around the point $p \in D$ by

$w(x, y):=w_{1}(x, y)+w_{2}(x, y)+w_{3}(x, y), x, y \in D$.

Branching points are natural realizations of logical structures. As an example consider Figure 3. The figure shows path embeddings as blue lines between the black points. Three branching points can be identified.

Here, as a simple example and first step towards more complicated logic we formulate logical OR and multiplexer elements via branching points. A pulse corresponds to the logical input true or 1. No pulse corresponds to false or 0 .
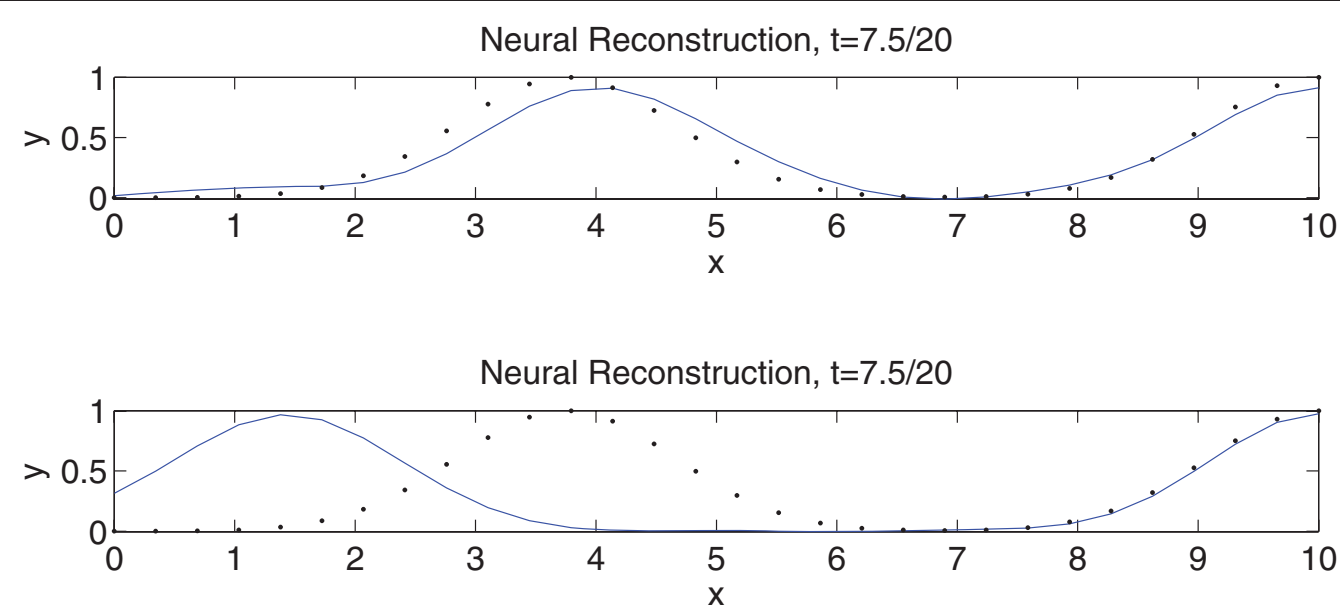

FIGURE 2 |We show the time-slice of a delay gate dynamics as described in Section “Embedding One-dimensional Processes into 2d or 3d Neural Tissue". Here, the delay gate is the complete interval between $x=0$ and 10 . In the upper graphics the pulse (blue line) entered the delay gate earlier than in the second image. Both pulses excite the exit area of the gate simultaneously. The dotted curve shows the original training patterns. For the generation of the neural kernel we used the inversion technique of Section "Solving Inverse Neural Field Problems". 


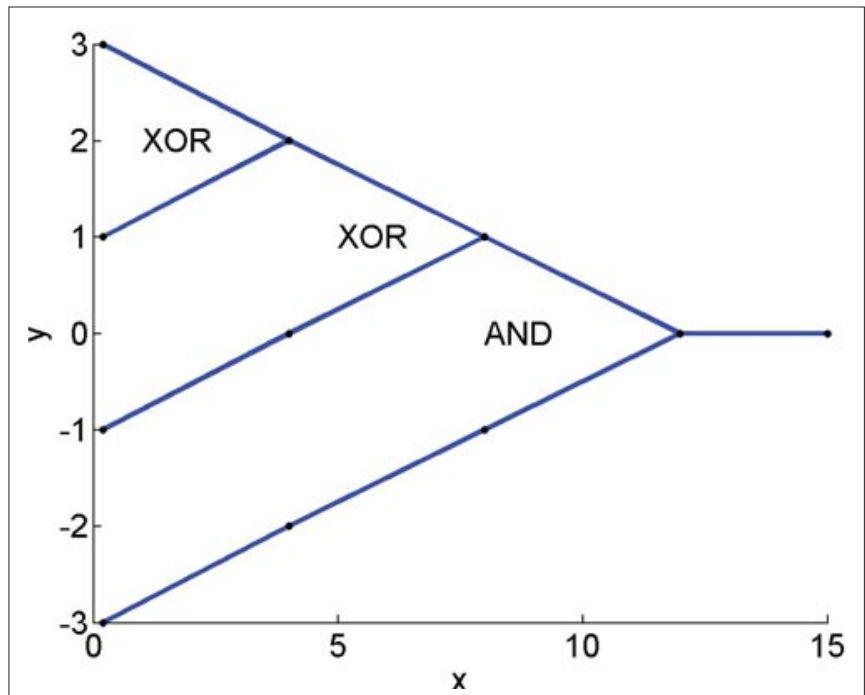

FIGURE 3 |A logical design for a gated binary addition, where here the last digit as defined in Eq. 35 is calculated.

Algorithm 3.3. OR Logic, Multiplexer Branching points where $w_{1}$ and $w_{2}$ are incoming and $w_{3}$ is outgoing implement the classical OR logic in the sense that a pulse is transmitted through $\Gamma_{3}$ if either a pulse enters the branching point through $\Gamma_{1}$ or through $\Gamma_{2}$ or both $\Gamma_{1}$ and $\Gamma_{2}$.

Branching points where $w_{1}$ is incoming and $w_{2}, w_{3}$ are outgoing implement a multiplexer logic in the sense that a pulse entering the branching point will be transmitted into both $w_{2}$ and $w_{3}$.

\section{LOGICAL ELEMENTS}

Further logical elements are now constructed using embedded pulses, branching points and additional inhibition elements.

An inhibition element is a neural kernel $w_{i}$ defined on two subsets $V_{1} \subset \Gamma_{1}$, and $V_{2} \subset \Gamma_{2}$, such that

$w_{i}(x, y):=\left\{\begin{array}{cc}-c_{-}, & x \in V_{2}, y \in V_{1} \\ 0, & \text { otherwise. }\end{array}\right.$

An inhibition element establishes an inhibition which is activated by a pulse travelling through $V_{1}$ and is applied to any field in $V_{2}$.

We are now prepared to define all standard logical elements within the neural field environment. Here, we pick selected examples.

XOR Logic. Consider three points $p_{\text {in }, 1}, p_{\text {in, } 2}$ and $p_{\text {out }} \in D$ in a neural tissue. We consider $p_{\mathrm{in}, 1}$ and $p_{\mathrm{in}, 2}$ as input nodes and $p_{\text {out }}$ as output node for logical processing, i.e. we expect input pulses in a neighbourhood $B_{\rho}\left(p_{\text {in, } 1}\right)$ or $B_{\rho}\left(p_{\text {in, } 2}\right)$ of these points as input. The output will be a field activation $u$ which is above the threshold $\eta$ on $B_{\rho}\left(p_{\text {out }}\right)$. We need to assume that

$d\left(p_{\text {in }, 1}, p_{\text {in }, 2}\right), d\left(p_{\text {in }, 1}, p_{\text {out }}\right), d\left(p_{\text {in }, 2}, p_{\text {out }}\right)>>\rho$

where $d(p, q)$ denotes the Euclidean distance between points $p$ and $q$ in $D$.

1. Let $w_{\text {plain, } \xi}$ be an embedded kernel for a plain pulse from $p_{\text {in, } \xi}$ to $p_{\text {out }}$ along a path $\Gamma_{\xi}$, where the tube $\Gamma_{\xi, p}$ has distance larger than $\rho$ to the point $p_{\text {in, } \eta}$ for $\eta \neq \xi, \eta, \xi=1,2$. We assume that the tubes $\Gamma_{1, \rho}$ and $\Gamma_{2, \rho}$ intersect only on the exit area around $p_{\text {out }}$.

2. Let $w_{i, \xi}$ be an inhibition kernel for the subsets $\Gamma_{\xi, \rho} \mid \mathrm{V}$ and $\Gamma_{\eta, \rho} \mid \mathrm{V}$ with $V:=\Gamma_{\xi, \rho} \cap \Gamma_{\eta, \rho}$ for $\xi \neq \eta, \xi, \eta=1,2$, i.e. $w_{\mathrm{i}, \xi}$ establishes an inhibition of fields in $\Gamma_{\eta, \rho} \backslash \mathrm{V}$ when a pulse in the tube area $\Gamma_{\xi, \rho} \mid \mathrm{V}$ is active.

If the constants are chosen appropriately, the above setting realizes a logical XOR functionality.

Algorithm 3.4. XOR Logic. The kernel

$w(x, y):=$

$w_{\text {plain }, 1}(x, y)+w_{\text {plain }, 2}(x, y)+w_{i, 1}(x, y)+w_{i, 2}(x, y), x, y \in D$

establishes a XOR functionality for pulses entering the tubes $\Gamma_{1}$ and $\Gamma_{2}$ connecting $p_{\text {in, } 1}$ and $p_{\text {in, } 2}$ with $p_{\text {out }}$. The functionality will be satisfied for pulses entering the paths $\Gamma_{1}$ and $\Gamma_{2}$ at any point and during the time window defined by the remaining travel time of a pulse through these paths.

We have numerically tested the above scheme independently and for realizing the binary addition logic described in Section "An Abstract View and Basis Transformations" and shown in Figure 5, where traces of time-dependent travelling pulses are shown. The underlying logical structure is visualized in Figure 3. We assume that an input excitation is given to the input nodes at $(0,-1),(0,1)$ and $(0,3)$, the node $(0,-3)$ serves as a gating pulse to activate the complete logical gate. As before, a pulse corresponds to the logical input true or 1 . No pulse corresponds to false or 0 . The inhibition components lead to mutual inhibition between the pulses in the paths of the XOR elements.

Here we have combined analytical parts (the inhibition elements are explicitly prescribed) with results of the inversion of Section "Solving Inverse Neural Field Problems" for obtaining travelling pulses in the lines between the nodes. The elements are constructed in one dimension and then embedded into the two-dimensional neural domain by the embedding technique of Definition 3.1.

AND Logic. There are at least two basic options to realize the classical AND logic for pulses. The first option combines the OR logic given by Algorithm 3 with a damping of the kernels in the overlap of the exit point.

Algorithm 3.5. AND Logic I. Let $w_{\text {or }}$ be the kernel constructed in Algorithm 3 and $V:=\Gamma_{\xi, \rho} \cap \Gamma_{\eta, \rho}$. We define a damped kernel by

$w_{\text {and }}(x, y):=\left\{\begin{array}{cc}c_{d} \cdot w_{\text {or }}(x, y) & x \in V \\ w_{\text {or }}(x, y) & \text { otherwise. }\end{array}\right.$

with damping factor $c_{\mathrm{d}} \in(0,1)$. If the damping factor is chosen appropriately (depending on $\rho$ and the kernel setup), then $w_{\text {and }}$ establishes the AND functionality for pulses entering $\Gamma_{1}$ and $\Gamma_{2}$. The time window for synchronization here is rather short, since both pulses need to arrive at $\rho_{\text {out }}$ simultaneously. This can be enhanced by using delay gates in both of the tubes which are involved.

An alternative implementation of AND is provided by the following architecture, visualized in Figure 4. 


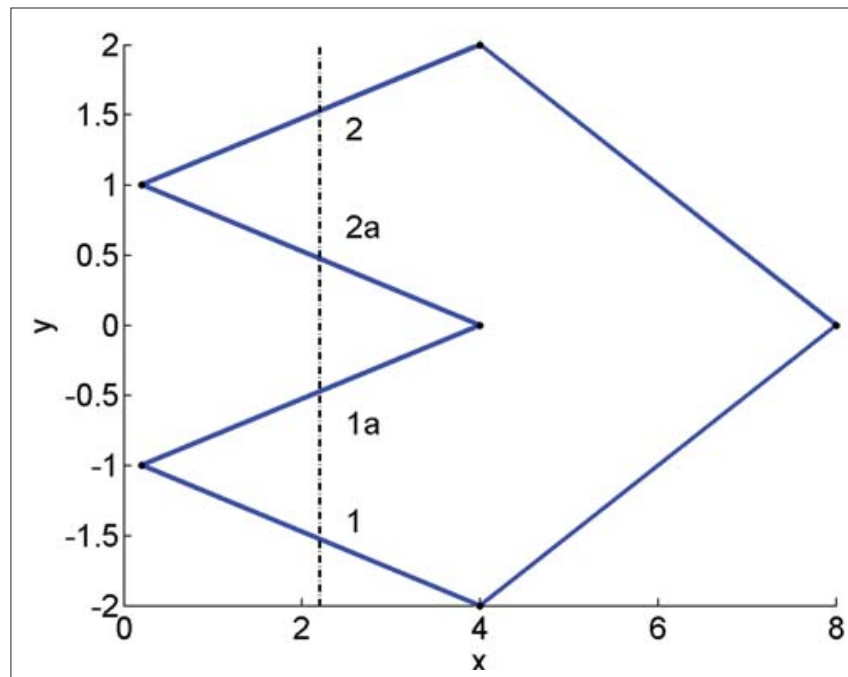

FIGURE 4 | A structural design for a stable logical AND gate. The dashed line separates different parts of the logical paths for 1, 2, 1a and 2a where different inhibition effects are employed, details are introduced in the points $1-4$ in Algorithm 3.6. Pulses enter at the nodes in $(0,-1)$ and $(0,1)$. The output node is at $(8,0)$.

Algorithm 3.6. AND Logic II. We construct path embeddings as visualized in Figure 4 with the following path kernels and inhibitions:

1. From two input nodes we construct two paths each by a multiplexer branching point according to Algorithm 3.3. This leads to four paths labelled 1, 1a, 2 and $2 \mathrm{a}$. We use the numbers as abbreviations for either paths or tubes.

2. We now apply inhibition from 1 to $2 \mathrm{a}$ and from 2 to $1 \mathrm{a}$ on the first half of the paths.

3. After that in the second half of the path we apply inhibition from 1 a to 1 and from 2 a to 2 .

4. Then, we connect 1 and 2 to the endpoint by a simple OR gate.

The combination of the steps 1 to 4 generates a logical AND gate embedded into neural tissue.

To show the validity of the above setup we need to check four situations, with no pulses or with pulses entering in either $p_{\text {in, } 1}, p_{\text {in, } 2}$ or in both points. No pulse represents $0 \& 0=0$, which is clearly satisfied. Consider the situation $1 \& 0$. In this case we get pulses in 1 and $1 \mathrm{a}$. Then $1 \mathrm{a}$ inhibits the pulse in 1 such that it dies and there is no output pulse, i.e. $1 \& 0=0$ is satisfied. The same logic applies to the pulses in 2 and $2 \mathrm{a}$. Now, consider two pulses $1 \& 1$. Then first they split into four pulses $1,1 a, 2,2 a$. Now we apply step 2 , such that the pulses in $1 a$ and $2 a$ die by inhibition from 2 and 1 . Then the remaining pulses in 1 and 2 go through and via the OR gate reach the final point $p_{\text {out }}$, which establishes the logic $1 \& 1=1$.

The advantage of the AND logic of Algorithm 3.5 is that it is a very simple and natural implementation depending on the special size of the kernel $w$. The second implementation is much more independent of this size and much more stable with respect to strength variations of $w$. Both designs are implemented in a neural domain by the embedding techniques of Definition 3.1.

Binary Addition. Finally, we would like to show a more complex implementation of binary addition. Here, we have realized the addition of three binary numbers triggered by a gating pulse, i.e. we realize

a) $0+0+0=0, b) 1+0+0=1, c) 0+1+0=1, d) 0+0+1=1, e)$

$1+1+0=0, f) 1+0+1=0, g) 0+1+1=0, h) 1+1+1=1$. (35)

The logical design is summarized in Figure 3. The idea is that the pulses enter the neural domain at points $(0,-1),(0,1)$ and $(0,3)$, where we also added a gating pulse with entry at $(0,-3)$. We will study different solutions of the realization of the above logical structure. The first solution is a pulse-based approach where the pulses are constructed in one dimension first on the basis of Section "Solving Inverse Neural Field Problems". They are embedded into a two-dimensional neural domain, combined with inhibition elements, glued together at branching points or simple connection points. The second and third approach will be described in the following sections.

In Figure 5 we show the trace of the pulses - not their timebehaviour, i.e. we show all points red where the pulse is above the threshold at some point of time. The binary inputs are the first three input nodes on the left-hand side of the tissue, the output node is the centred node on the right-hand side.

\section{EMBEDDING OD LOGIC INTO NEURAL TISSUE}

Our approach here to a OD embedding is the reduction of neural pulse dynamics. We consider the neural tissue $D$ to be global in the sense that every point $x$ can be influenced by a neural field at the point $y \in D$. But this means that we do not need to process an influence by a travelling pulse, but we can directly excite the corresponding areas in a non-local fashion.

In contrast to a path embedding, here we need only to embed two or more points with their neighbourhoods into the neural tissue to realize a logical function. Since a point is a zero-dimensional manifold, we call the embedding zero-dimensional (0D).

The implementation of such $0 \mathrm{D}$ embeddings reflects old issues of implementing logical functions in neural networks (Hertz et al., 1991). For example, a logical XOR functionality cannot be implemented by directly linking two input nodes to the output node. We need to include at least two further points with their neighbourhoods.

With the neural field we have a highly integrated continuous auto-associative network in which we may embed classical neural network logic. But we also gain new options for these embeddings and the deep tools of mathematical analysis and functional analysis are available to investigate and control such embeddings. These further options will be explored in our final section, here we will provide more details on the $0 \mathrm{D}$ embedding.

Pulses. Zero-dimensional versions of pulses are direct excitations of the target areas within the neural tissue. To obtain a speed of pulse propagation comparable to the speed of elementary logical elements we have used a one-stop pulse realization, i.e. from an area around an input point $p_{\text {in }}$ we have first excited an auxiliary area around a point $p_{\text {aux }}$ and then this area excites the target area around $p_{\text {out }}$.

XOR Logic. For the XOR gate we have used a zero-dimensional logic with five points, two input points $p_{\text {in, } 1}, p_{\text {in, } 2}$, two auxiliary points $p_{\text {aux }, 1}, p_{\text {aux }, 2}$ and the output point $p_{\text {out }}$. Excitation is taking place from $p_{\text {in }, \xi}$ to $p_{\text {aux }, \xi}$ and from $p_{\text {aux }, \xi}$ to $p_{\text {out }}$ for $\xi=1,2$. Inhibition is realized from $p_{\text {in }, \xi}$ to $p_{\text {aux }, \eta}$ with $\xi=\eta, \xi, \eta=1,2$.

AND Logic. For the AND gate as for XOR we have used a zerodimensional logic with six points, two input points $p_{\text {in, },}, p_{\text {in }, 2}$, three 

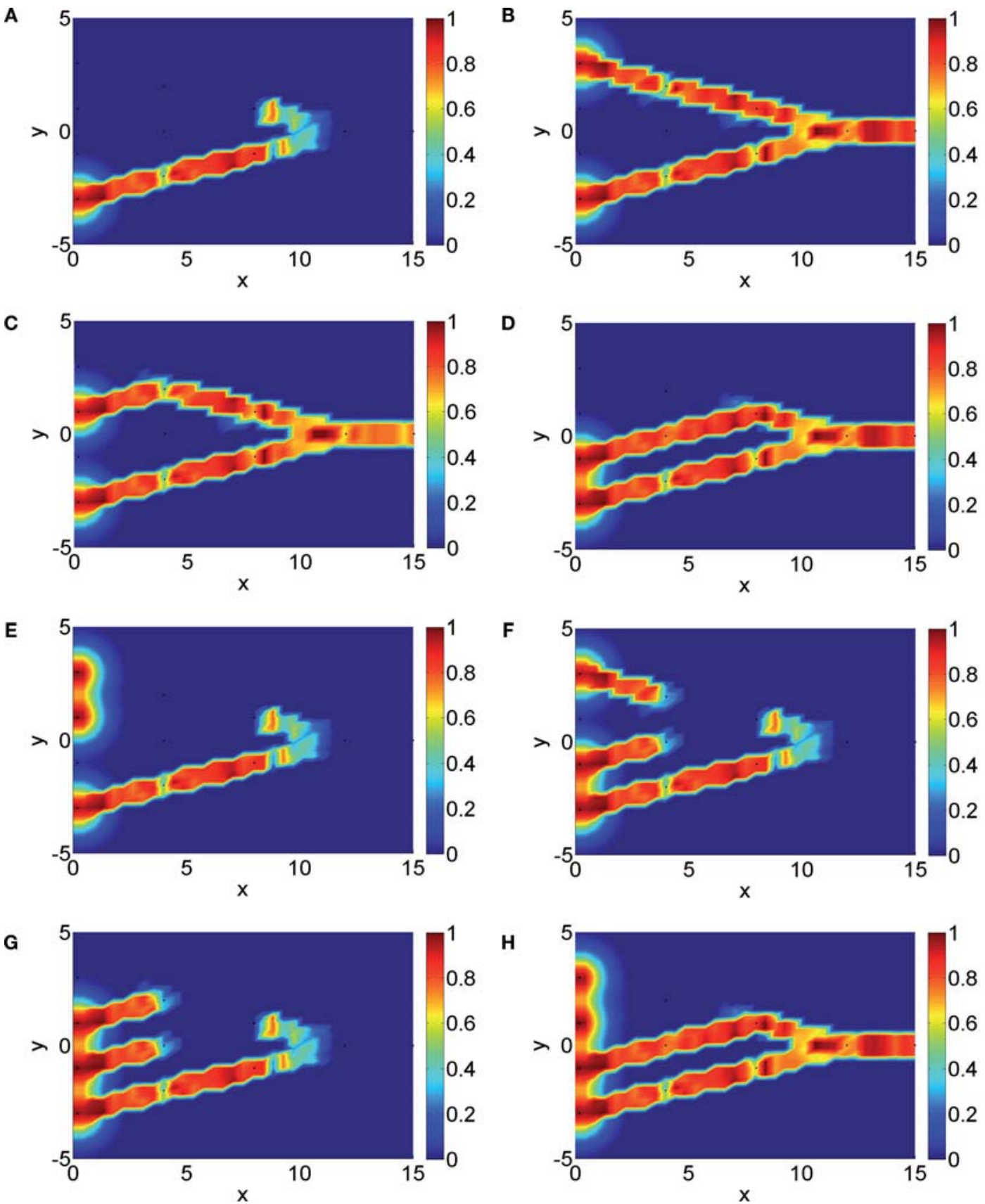

FIGURE 5 |The images show the traces of neural pulses for the last binary digit of a gated binary addition logic (Eq. 35a-h) corresponding to the panels A-H via the one-dimensional embedding technique in combination with low-dimensional inverse problems and analytical elements for the logical components.

auxiliary points $p_{\text {aux }, 1}, p_{\text {aux }, 2}, p_{\text {aux }, 3}$ and the output point, $p_{\text {out }}$. Excitation is taking place from $p_{\mathrm{in}, \xi}$ to $p_{\mathrm{aux}, \xi}$ for $\xi=1,2$, from $p_{\mathrm{in}, \xi}$ to $p_{\mathrm{aux}, 3}$ and from $p_{\text {aux }, \xi}$ to $p_{\text {out }}$ for $\xi=1,2$. Inhibition is realized from $p_{\text {aux }, 3}$ to $p_{\text {out }}$. Here, a single input in either $p_{\mathrm{in}, 1}$ or $p_{\mathrm{in}, 2}$ will excite either $p_{\mathrm{aux}, 1}, p_{\mathrm{aux}, 3}$ or $p_{\text {aux }, 2}, p_{\text {aux }, 3}$. Then the inhibition of $p_{\mathrm{aux}, 3}$ to $p_{\text {out }}$ will cancel the excitation if there are not two simultaneous excitations from $p_{\text {aux }, 1}$ and $p_{\text {aux }, 2}$.

In the results of Figure 6 the auxiliary points have been inserted into the path between the input and output nodes. As in Figure 5 here we show the trace of the pulse, not its time-behaviour, i.e. we show all points red where the pulse is above the threshold at some point of time.

Binary Addition. Finally, we have integrated the zero-dimensional embeddings into a binary addition logic. It is shown in the results of Figure 6. The design is analogous to the one shown in Figure 3.

\section{AN ABSTRACT VIEW AND BASIS TRANSFORMATIONS}

The goal of this section is to formulate an abstract view into state space dynamics of logical computations. Logical dynamics 


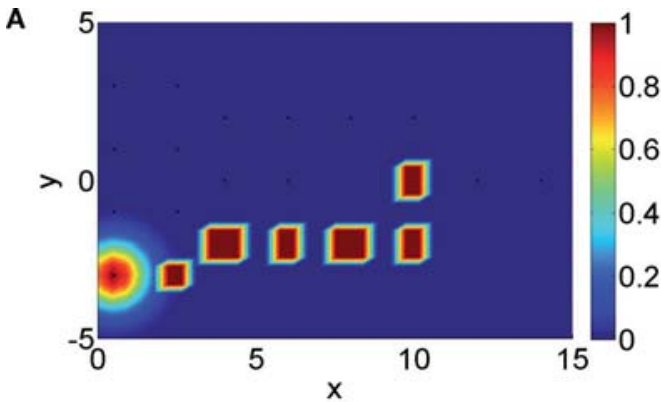

C

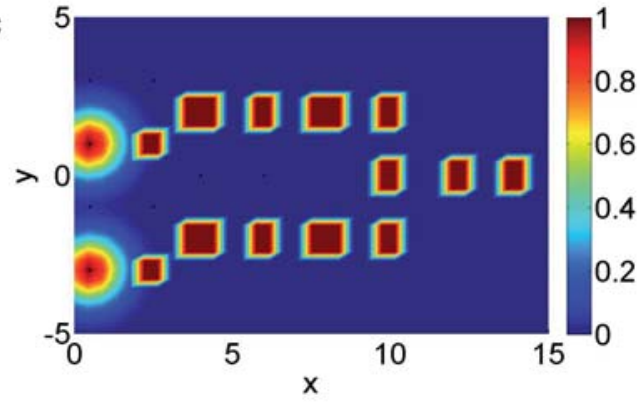

E

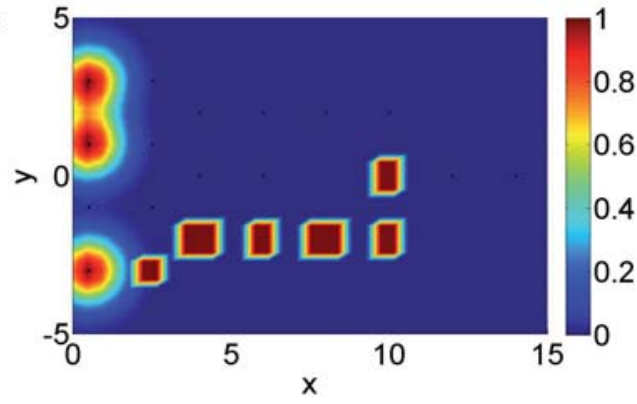

G

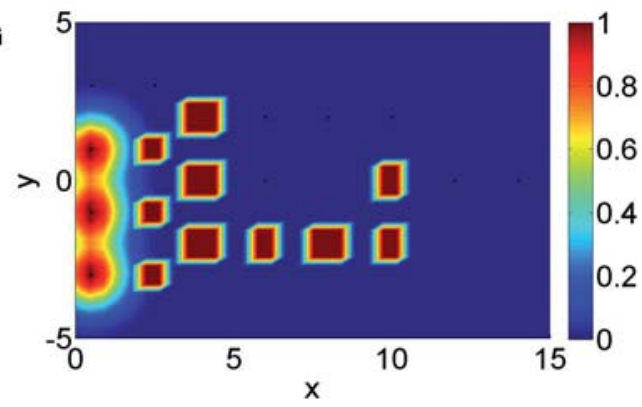

B

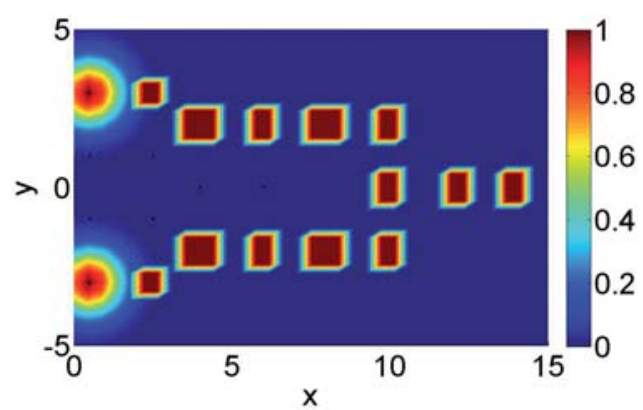

D

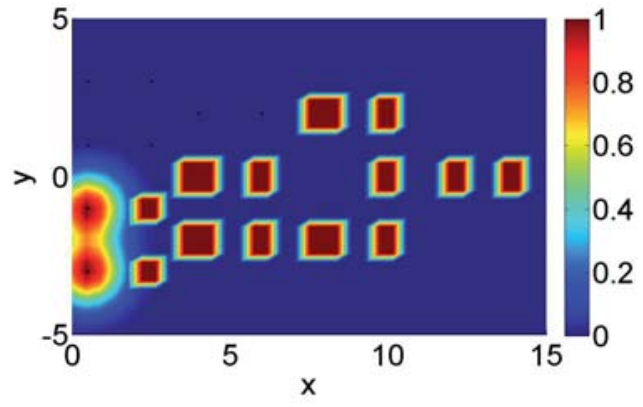

$\mathbf{F}$

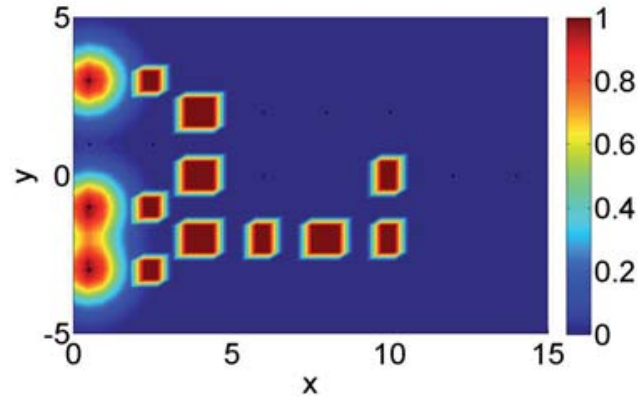

H

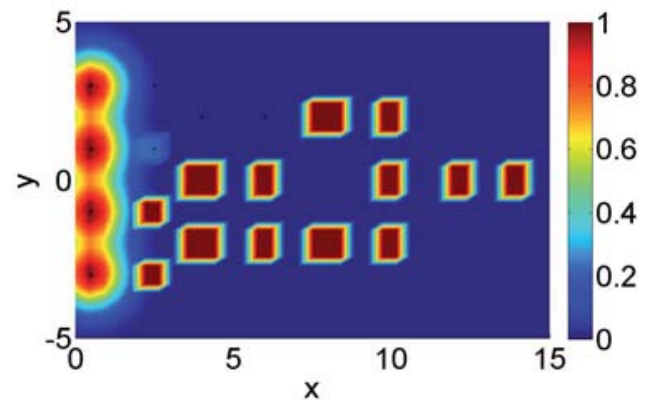

FIGURE 6 |The images show the traces of neural pulses for the last binary digit of a gated binary addition logic (Eq. 35a-h) corresponding to the panels A-H via the zero-dimensional embedding technique with purely analytical elements for the logical components, compare Section "Embedding OD Logic into Neural Tissue".

is often regarded as processing from one logical state to another (Wennekers, 2006, 2007; beim Graben and Potthast, 2009). Here, we will construct particular logical states and neural mappings from one state to another using the zero-dimensional embeddings from Section "Embedding 0D Logic into Neural Tissue" and Hilbert space basis transformations. Thus, we stably construct complex distributed state-space dynamics for neural processes.

\section{HILBERT SPACE DYNAMICS}

We aim to discuss a Hilbert space dynamical implementation of logical structures. Let $X$ be some Hilbert space.
Definition 5.1. Let $X$ be a Hilbert space and consider a parameterized mapping

$S_{t}: X \rightarrow X, t>0$,

with the property

$S_{t+s}=S_{t} \circ S_{s}$

for all $t, s>0$. A mapping of the type (Eq. 36), (Eq. 37) establishes a Hilbert space dynamics.

Remark. Given an initial value $u_{0} \in X$, the dynamics $S_{t}$ defines a time-dependent field 
$u_{t}:=S_{t} u_{0}, t \geq 0$

with the property

$u_{t+s}=S_{s} u_{t} s, t \geq 0$

i.e. the operator $S_{s}$ describes the change of the field $u_{t}$ from $t$ to $t+s$. This is well-known as semi-group representation in the mathematical theory of dynamical systems (Anosov and Arnol'd, 1988). Here, it establishes an abstract concept which is realized by the Amari neural field equation 2 .

We can discretize the dynamics by considering

$t_{k}=k \cdot h, k=0,1,2, \ldots$

with some grid constant $h_{t}$. Then, according to Eq. 37 the complete dynamics is given by iterating $S:=S_{h_{t}}$.

We consider elements $p_{k}^{(j)} \in X$ for $k=1, \ldots, n$ and $j=1, \ldots, m$. Our goal here is the construction of particular dynamical systems which satisfies

$p_{k+1}^{(j)}=S\left(p_{k}^{(j)}\right), k=1, \ldots, n-1$,

with initial values $p_{1}^{(j)} \in X$ for $j=1, \ldots, m$. For fixed $j$ the sequence of points $p_{k}^{(j)}, k=1, \ldots, n$ is a realization of a particular part of logical inference.

The dynamics under consideration in Eq. 41 in general will not be linear, but rather highly nonlinear in most cases. For example, consider the space $\mathbb{R}^{3}$ and initial elements

$p_{1}^{(1)}:=(1,0,0), p_{1}^{(2)}:=(0,1,0), p_{1}^{(3)}:=(1,1,0)$.

If we define the second element as

$p_{2}^{(1)}:=(0,0,1), p_{2}^{(2)}:=(0,0,1), p_{2}^{(3)}:=(0,0,0)$

we have defined the classical logical XOR function in the third component, i.e. $(1,0)$ is mapped onto $1,(0,1)$ is mapped onto 1 , but $(1,1)$ is mapped onto 0 .

The above implementation of a logical XOR gate can be carried out in an abstract setting with a general set of Hilbert space elements. Here, we will realize this on the basis of the above logical implementations by a basis transformation of a subspace. This will be explained in our next subsection.

\section{BASIS TRANSFORMATIONS, PERMUTATION MATRICES AND NEURAL DYNAMICS}

A basis transformation is an injective mapping $T$ of a basis $\mathcal{B}_{1}:=\left\{g_{1}, \ldots, g_{n}\right\}$ of a space $X$ onto a basis $\mathcal{B}_{2}:=\left\{f_{1}, \ldots, f_{n}\right\}$ of $X$ with $T g_{k}=f_{k}, k=1, \ldots, n$. Here, for simplicity we will restrict our attention to basis transformations of finite dimensional spaces. But all arguments will generalize to an infinite dimensional setting. The mapping $T$ defines a one-to-one mapping between $\mathcal{B}_{1}$ and $\mathcal{B}_{2}$, and via this mapping a one-to-one mapping of $X$ onto itself given by

$T \varphi=T\left(\sum_{k=1}^{n} \alpha_{k} g_{k}\right)=\sum_{k=1}^{n} \alpha_{k}\left(T g_{k}\right)=\sum_{k=1}^{n} \alpha_{k} f_{l}$

where an element $\varphi$ is expressed in terms of the basis elements by

$\varphi=\sum_{k=1}^{n} \alpha_{k} g_{k}$, with $\alpha_{k} \in \mathbb{R}, k=1, \ldots, n$.
Consider a set of points $p_{1}, \ldots, p_{N}$ representing a discretization of some neural tissue. Then we obtain a Haar basis by setting

$u_{k}(x)= \begin{cases}1, & x=p_{k} \\ 0, & x \neq p_{k}\end{cases}$

for $x \in\left\{p_{1}, \ldots, p_{N}\right\}$ and $k=1, \ldots, N$.

Simple Example: Permutations. Any permutation $\Pi:\{1, \ldots, N\}$ $\rightarrow\{1, \ldots, N\}$ now defines a basis transformation by

$q_{k}=\Pi\left(p_{k}\right), k=1, \ldots, N$

Permutations are one-to-one by definition, thus we have an equivalent representation of any function in the new basis.

We can realize a permutation-based basis transformation by a permutation matrix, i.e. a matrix $\Pi$ which has exactly one entry 1 in each column and row. Given a vector $\mathbf{u}$ representing a neural field at point $t$ in time, we obtain its representation in another basis by $\Pi \mathbf{u}$.

General Case. Now we study the general case of basis transformations applied to a neural domain $D$. The discretization of the transformation operator $T$ from Eq. 44 will lead to a transformation matrix $\mathbf{T}$, which we express in terms of the Haar basis (Eq. 46), i.e. a basis function $g_{k}$ is given by a vector $\mathbf{g}_{k}$, the new basis $f_{k}$ is expressed as a vector $\mathbf{f}_{k}$ and $\mathbf{T}$ is a matrix such that

$\mathbf{T g}_{k}=\mathbf{f}_{k}$ for $k=1, \ldots, n$.

With the sigmoidal function $f$ defined in Eq. 3 and time steps defined in Eq. 40 the discretized Amari dynamics via a finitedifference method leads to a nonlinear state transition mapping given in the form

$\mathbf{u}_{k+1}=c \mathbf{u}_{k}+\mathbf{W} f\left(\mathbf{u}_{k}\right), k=1,2,3, \ldots$

with initial state $\mathbf{u}_{1}$ and some constant $c$. Some basis transformation $\mathbf{T}$ defines a transformed state dynamics $\mathbf{v}_{\mathrm{k}}=T \mathbf{u}_{k}$ for $k=1,2, \ldots$, with invertible matrix $\mathbf{T}$. Then, the transformed dynamics is given by

$$
\begin{aligned}
\mathbf{v}_{k+1}=\mathbf{T} \mathbf{u}_{k+1} & =c \mathbf{T} \mathbf{u}_{k}+\mathbf{T W} f\left(\mathbf{u}_{k}\right) \\
& =c \mathbf{T T}^{-1} \mathbf{v}_{k}+\mathbf{T W} f\left(\mathbf{T}^{-1} \mathbf{v}_{k}\right) \\
& =c \mathbf{v}_{k}+\mathbf{T W} f\left(\mathbf{T}^{-1} \mathbf{v}_{k}\right), \mathrm{k}=1,2, \ldots
\end{aligned}
$$

We write the transformed dynamics as Amari type state dynamics with transformed transition kernel $\tilde{\text { W }}$

$\mathrm{v}_{k+1}=c \mathrm{v}_{k}+\tilde{\mathrm{W}} f\left(\mathrm{v}_{k}\right), k=1,2, \ldots$

Thus, the transformation of the kernel $\mathbf{W}$ into $\tilde{\mathbf{W}}$ is given by

$\tilde{\mathrm{W}}=\mathrm{TW} \Phi \tilde{\Phi}^{\dagger}$

where $\Phi$ and $\tilde{\Phi}$ are given as in Eq. 9 by

$\Phi:=\left(f\left(\mathrm{u}_{k}\right)\right)_{k=1, \ldots, n}, \tilde{\Phi}:=\left(f\left(\mathrm{v}_{k}\right)\right)_{k=1, \ldots, n}$

and $\tilde{\Phi}^{\dagger}$ denotes the pseudo-inverse of $\tilde{\Phi}$. The arguments to derive the transformed dynamics are valid if $\tilde{\Phi}$ has a pseudo-inverse, which corresponds to our general condition that the matrix $\Phi$ has full rank, compare Eq. 9. Note that in the special case where $f(x)=x$ and the matrices are square we obtain $\Phi \tilde{\Phi}^{\dagger}=\mathrm{T}^{-1}$, i.e. then we have $\tilde{\mathrm{W}}=\mathrm{TWT}^{-1}$. In general, Eq. 52 provides a transformation of the Hebb rule for constructing neural field kernels (Potthast and beim Graben, in press). 


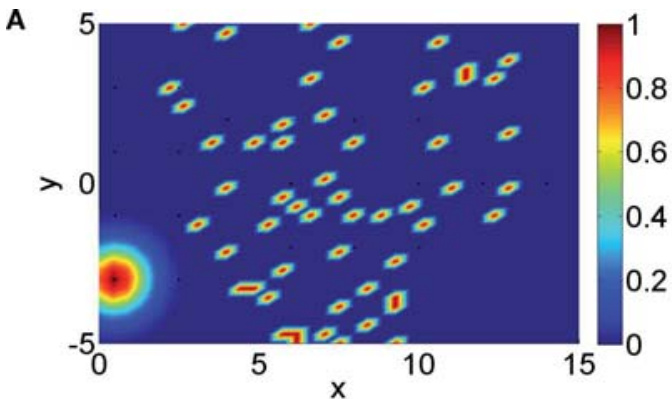

C

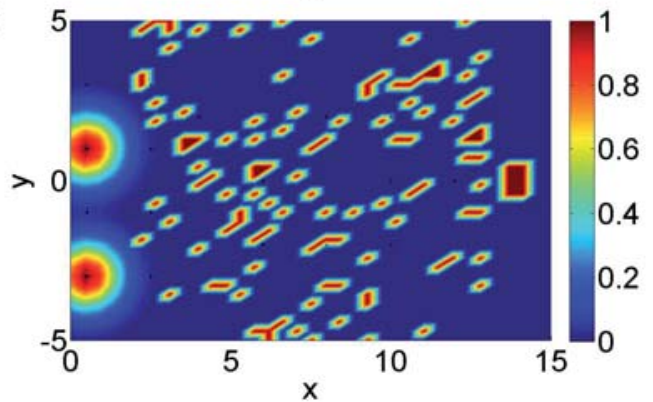

E

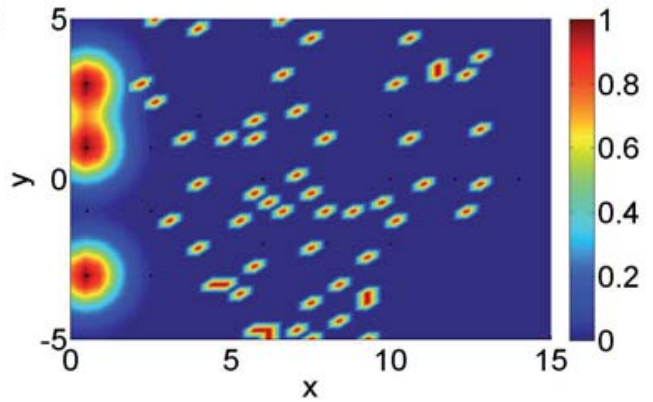

G

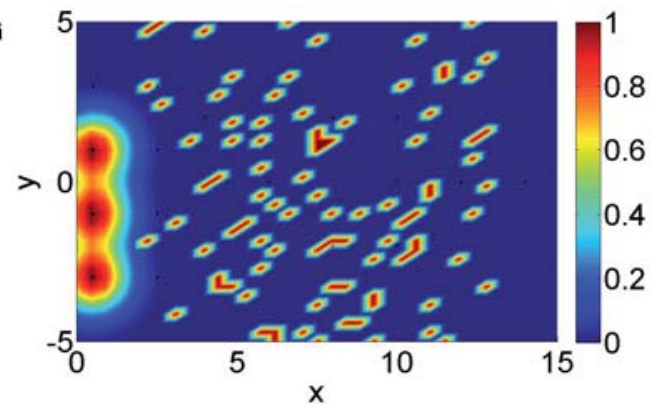

B

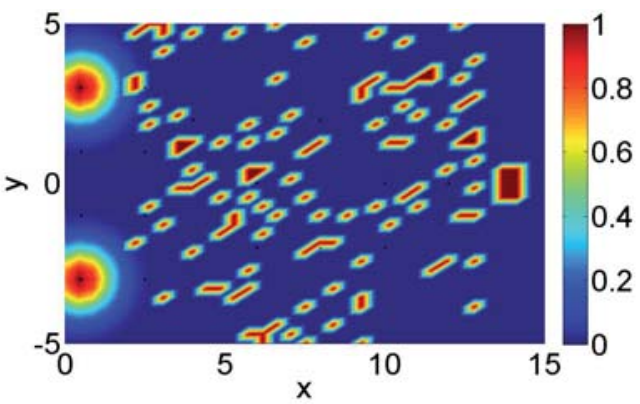

D
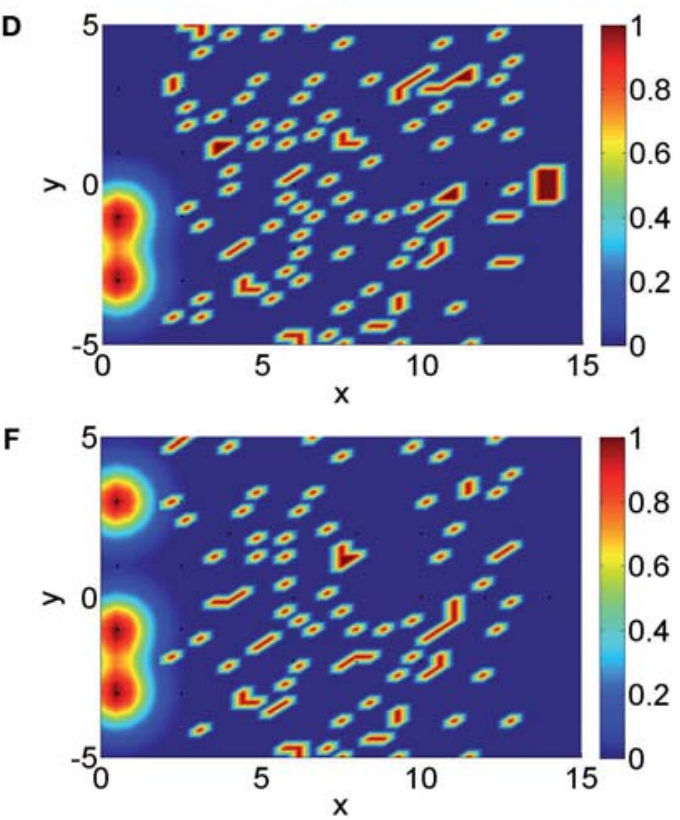

H

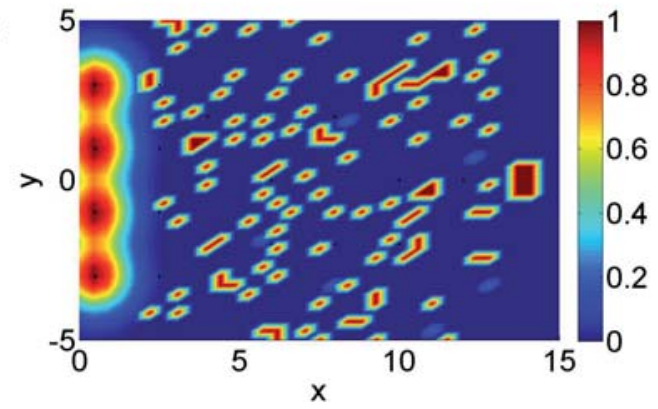

FIGURE 7 |The images show the traces of neural pulses for the last binary digit of a gated binary addition logic (Eq. 35a-h) corresponding to the panels A-H via the basis transformation technique of Section "An Abstract View and Basis Transformations" in combination with the zero-dimensional embedding technique with purely analytical elements for the logical components of Section "Embedding 0D Logic into NeuralTissue".

Equation 52 provides a simple approach to constructing complicated state space dynamics for neural domains based on the Amari equation from some simpler dynamics given by $\mathbf{W}$. If we combine it with the lowdimensional embedding techniques from previous sections we obtain a flexible and powerful tool to construct global neural kernels.

\section{DISTRIBUTED LOGICAL DYNAMICS}

We will use a basis generated by random vectors to obtain distributed logical processing which establishes a state dynamics in a neural Hilbert space.
Algorithm 5.2. Complex Distributed Logic. A distributed statespace dynamics realizing the binary addition dynamics (Eq. 35) as designed in Sections "Embedding One-dimensional Processes into $2 \mathrm{~d}$ or $3 \mathrm{~d}$ Neural Tissue" and "Embedding 0D Logic into Neural Tissue", compare also Figures $\mathbf{5}$ or $\mathbf{6}$, is constructed by the following steps.

1. We first construct a stable and simple zero-dimensional logical kernel W representing the binary dynamics (Eq. 35) visualized in Figure 3. 

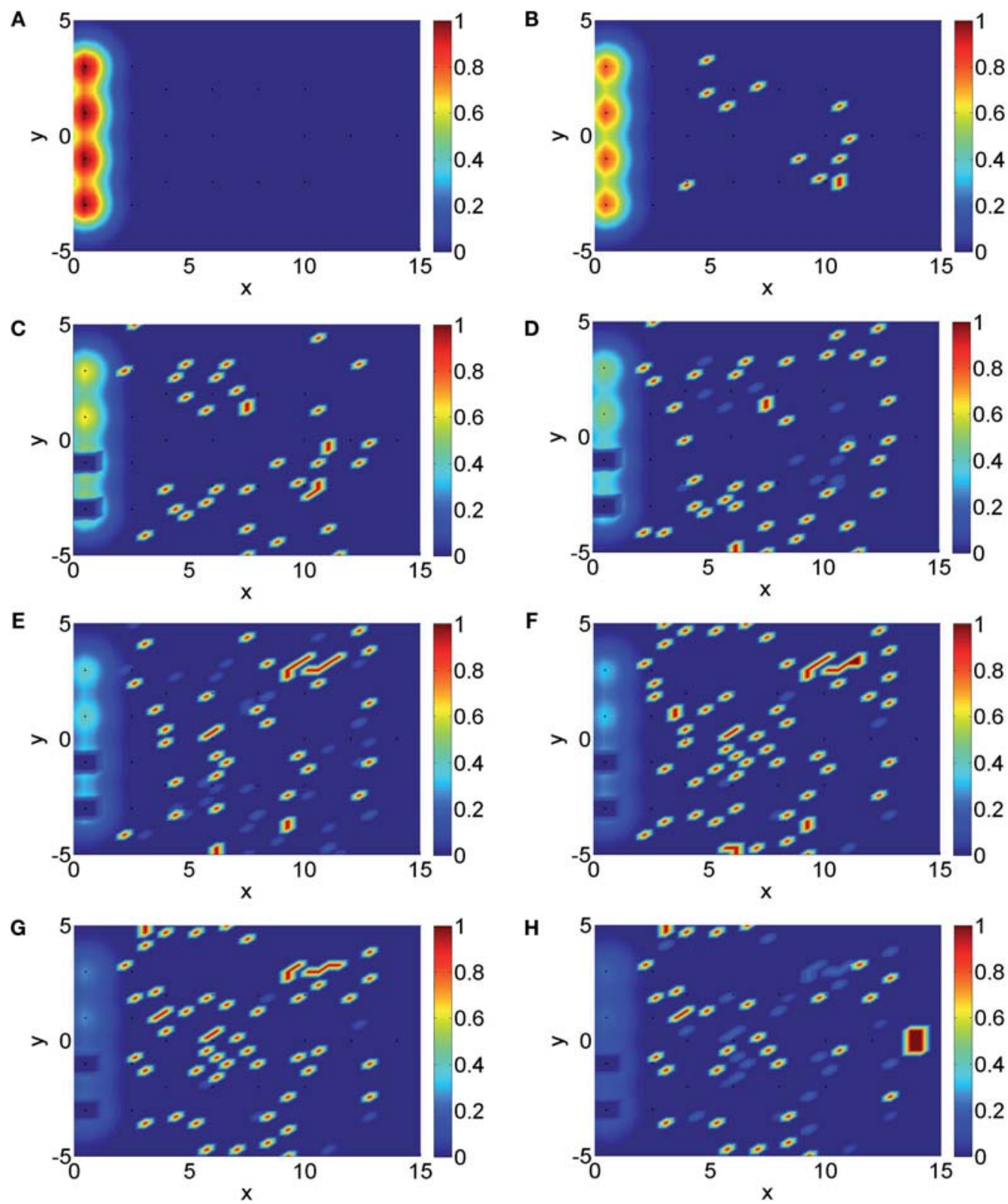

FIGURE 8 |We illustrate the time dynamics of the distributed processing of $1+1+1=1$ with gating pulse, displaying the neural field at eight time steps (Eq. 54) for $\mathbf{k}=\mathbf{1}$ for panel A, $\mathbf{k}=\mathbf{2}$ for panel $\mathrm{B}, \mathbf{k}=\mathbf{3}$ for panel $\mathbf{C}, \mathbf{k}=\mathbf{4}$ for panel $\mathbf{D}, \mathbf{k}=\mathbf{5}$ for panel $\mathbf{E}, \mathbf{k}=\mathbf{6}$ for panel $\mathrm{F}, \mathbf{k}=\mathbf{7}$ for panel $\mathbf{G}, \mathbf{k}=\mathbf{8}$ for panel $\mathbf{H}$. The fields seem to show a random flickering due to the distributed nature of the basis activated basis elements. However, the binary logic is fully implemented in the distributed neural field dynamics and here leads to the desired excitation of the exit node as a result of the neural processing.

2. Then, we use a basis transformation $\mathbf{T}$ based on the mapping of our original basis functions onto a set $\left\{\mathbf{v}_{k}, k=1,2, \ldots, \mathrm{n}\right\}$ of states which have been generated by a random set of ones and zeros in our discretized neural domain. Here, we kept the input nodes and output node intact. The transformed kernel $\tilde{\mathbf{W}}$ is defined by Eq. 52 .

We show the traces of this distributed logical processing in Figure 7. The logic is now realized by the excitation and inhibition of some general Hilbert space elements which are distributed randomly over the full neural tissue.
The time dynamics of these fields is illustrated in Figure 8, where in a), b), c) ,..,h) we show snapshots of the fields at points $t_{k}$ in time for

$k=m \cdot k_{0}, m=1, \ldots, 8$

with some appropriate constant $k_{0} \in \mathbb{N}$. It seems to show a random ("flickering") dynamics of neural fields. But the logic which is implemented here is deterministic and according to the transformation (Eq. 52) equivalent to the pulse logic and zero-dimensional logic in the Figures 5 or 6. 


\section{CONCLUSIONS}

We have shown that neural field kernels can be constructed in a stable and computationally cheap way to carry out complex cognitive tasks using embedding techniques which map zero- or one-dimensional (computational or analytical) solutions into a higher-dimensional neural space.

Further, we have shown a variety of different implementations of complex logical tasks like binary addition with several input nodes. In particular, we have shown that we can achieve a stable and controllable

a) pulse-dynamics,

b) localized state dynamics and

c) distributed Hilbert space-based logical dynamics

in a neural field environment. This provides a basis for the further study of artificial or natural cognitive dynamics based on continuous connectionist structures.

For the one-dimensional embedding, we constructed local kernels for describing pulses that travel along directed continuous paths in the neural domain. This is essentially a continuous version of classical connectionist feed-forward architectures.

\section{REFERENCES}

Amari, S.-I. (1977). Dynamics of pattern formation in lateral-inhibition type neural fields. Biol. Cybern. 27, 77-87.

Anosov, D. V., and Arnol'd, V. I. (eds) (1988). Dynamical Systems. Berlin, Springer-Verlag.

Balkenius, C., and Gärdenfors, P. (1991). Nonmonotonic inferences in neural networks. In Principles of Knowledge Representation and Reasoning, J. A. Allan, R. Fikes and E. Sandewall, eds (San Mateo, CA, Morgan Kaufmann), pp. 32-39.

Basar, E. (1998). Brain Function and Oscillations, Vol. I: Brain Oscillations. Principles and Approaches. Berlin, Springer-Verlag.

Blutner, R. (2004). Nonmonotonic inferences and neural networks. Synthese $142,143-174$.

Bressloff, P. C., and Cowan, J. D. (2002). The visual cortex as a crystal. Physica D 173, 226-258.

Colton, D., and Kress, R. (1998). Inverse Acoustic and Electromagnetic Scattering Theory. Berlin, Springer-Verlag.

Coombes, S., Lord, G., and Owen, M. (2003). Waves and bumps in neuronal networks with axo-dendritic synaptic interactions. Physica D 178, 219-241.

Erlhagen, W., and Schöner, G. (2002). Dynamic field theory of movement preparation. Psychol. Rev. 109, 545-572.

Ermentrout, G. B., and McLeod, J. B. (1993). Existence and uniqueness of travelling waves for a neural network. Proc. R. Soc. Edinb. 123A, 461-478.

beim Graben, P. (2008). Foundations of neurophysics. In Lectures in Supercomputational Neuroscience: Dynamics in Complex Brain Networks, P. beim Graben, C. Zhou, M. Thiel and J. Kurths, eds (Berlin, Springer-Verlag), pp. 3-48.

beim Graben, P., and Potthast, R. (2009). Inverse problems in dynamic cognitive modeling. Chaos 19, 015103.

Griffith, J.S. (1963). A field theory of neural nets: I. Derivation of field equations. Bull. Math. Biophys. 25, 111-120.

Hertz, J., Krogh, A., and Palmer, R. G. (1991). Introduction to the Theory of NeuralComputation.Cambridge, MA, Perseus Books.

Hutt, A., and Atay, F. M. (2006). Effects of distributed transmission speeds on propagating activity in neural populations. Phys. Rev. E 73, 021906.

Jirsa, V. K., and Haken, H. (1996). Field theory of electromagnetic brain activity. Phys. Rev. Lett. 77, 960-963.

Jirsa, V. K., and Haken, H. (1997). A derivation of a macroscopic field theory of the brain from the quasi-microscopic neural dynamics. Physica D 99, 503-526.

Jirsa, V. K., and Kelso, J. A. S. (2000). Spatiotemporal pattern formation in neural systems with heterogeneous connection topologies. Phys. Rev. E 62, 8462-8465.

Kress, R. (1989). Linear Integral Equations. Berlin, Springer-Verlag.

On the other hand, zero-dimensional embeddings combined with basis transformation techniques open a field for non-local neural field dynamics that has not been explored so far. In particular, the abstract Hilbert space representations could be useful for implementing even more complex tasks than logical computations in neural field models, for example dynamical language processing (Maye and Werning, 2007; Werning and Maye, 2007; beim Graben and Potthast, 2009).

The oscillatory patterns observed in our simulations also highlight the functional significance of cortical oscillations in general (Basar, 1998). Moreover, the chains of local random patches in the distributed dynamics that excite each other mutually can be regarded as operational cell assemblies as introduced by Wennekers $(2006,2007)$. Hence the embedding of low-dimensional solutions of the neural inverse problem by means of Hilbert space basis transformations provide a promising way for the implementation of such processing architectures.

\section{ACKNOWLEDGMENT}

This research has been supported by an EPSRC Bridging the Gaps Award in the UK under grant number EP/F033036/1.

Kress, R. (1999). Numerical Analysis. New York, Springer-Verlag.

Maye,A., andWerning, M. (2007).Neuronal synchronization: from dynamic feature binding to object representations. Chaos Complex. Lett. 2, 315-325.

McCulloch, W. S., and Pitts, W. (1943). A logical calculus of ideas immanent in nervous activity. Bull. Math. Biophys. 5, 115-133.

Mizraji, E., and Lin, J. (2001). Fuzzy decisions in modular neural networks. Int J. Bifurcat. Chaos 11, 155-167.

Nunez, P.L. (1995). Neocortical Dynamics and Human EEG Rhythms. New York, Oxford University Press.

Potthast, R., and beim Graben, P. (in press). Existence and properties of solutions for neural field equations. Math. Methods Appl. Sci.

Potthast, R., and beim Graben, P. (in press). Inverse problems in neural field theory. SIAM J. Appl. Dyn. Syst.

Robinson, P. A., Rennie, C. J., Wright, J. J., Bahramali, H., Gordon, E., and Rowe, D. L. (2001). Prediction of electroencephalic spectra from neurophysiology. Phys. Rev. E63, 021903.

Thelen, E., Schöner, G., Scheier, C., and Smith, L. B. (2001). The dynamics of embodiment: a field theory of infant perseverative reaching. Behav. Brain Sci. 24, 1-86.

Wennekers, T. (2006). Operational cell assemblies as a paradigm for braininspired future computing architectures. Neural Inf. Process. Lett. Rev. 10 135-145.
Wennekers, T. (2007). A cell assembly model for complex behaviour. Neurocomputing 70, 1988-1992.

Werning, M., and Maye, A. (2007). The cortical implementation of complex attribute and substance concepts: synchrony, frames, and hierarchical binding. Chaos Complex. Lett. 2, 435-452.

Wilson, H. R., and Cowan, J. D. (1973). A mathematical theory of the functional dynamics of cortical and thalamic nervous tissue. Kybernetik 13, 55-80.

Conflict of Interest Statement: The authors declare that the research was conducted in the absence of any commercial or financial relationships that could be construed as a potential conflict of interest.

Received:07 May 2009; paper pending published:03 June 2009; accepted:08 September 2009; published online: 08 October 2009. Citation: Potthast $R$ and beim Graben $P$ (2009) Dimensional reduction for the inverse problem of neural field theory. Front. Comput. Neurosci. 3:17. doi: 10.3389/neuro.10.017.2009

Copyright (c) 2009 Potthast and beim Graben. This is an open-access article subject to an exclusive license agreement between the authors and the Frontiers Research Foundation, which permits unrestricted use, distribution, and reproduction in any medium, provided the original authors and source are credited. 